\title{
A Compact, Low-Profile, Broadside Radiating Two-Element Huygens Dipole Array Facilitated by a Custom-Designed Decoupling Element
}

\author{
Ming-Chun Tang, Senior Member, IEEE, Xiaoming Chen, Student Member, IEEE, Ting Shi, Student \\ Member, IEEE, Hanwen Tu, Zhentian Wu, Student Member, IEEE, and Richard W. Ziolkowski, Life Fellow, IEEE
}

\begin{abstract}
A compact, low-profile, broadside radiating, twoelement Huygens dipole array is developed for in-band fullduplex (IBFD) applications. Each radiating element is a multilayer near-field resonant parasitic (NFRP) design that is electrically small with $k a=\mathbf{0 . 7 6}$ at its resonance frequency, $f_{0}=\mathbf{1 . 5 1 1}$ GHz. The center-to-center distance between the elements is only $0.3 \lambda_{0}$. The array's outstanding performance is facilitated by a custom-designed decoupling element. This specially engineered scatterer is printed on an additional layer and consists of a pair of meander-line resonators connected by a metallic strip. The overall height of the entire system is only $\lambda_{0} / 20.3$. The passive decoupling element operates as several electrically small electric dipoles whose scattered fields mitigate the interactions between the two radiators. It provides very high isolation levels between them and improves the overall directivity of the array. The optimized array was fabricated, assembled and tested. The measured results, in good agreement with their simulated values, confirm that the developed decoupling structure not only increases the peak isolation level from 14.4 to $50.4 \mathrm{~dB}$; but it also improves the peak realized gain in the broadside direction by 0.5 $\mathrm{dB}$ and the corresponding front-to-back ratio (FTBR) value by $14.4 \mathrm{~dB}$.
\end{abstract}

Index Terms-Antenna array, broadside radiating, decoupling elements, electrically small antennas, Huygens dipole antennas, mutual coupling.

\section{INTRODUCTION}

$\mathbf{H}$ UYGENS dipole antennas (HDAs) have drawn considerable attention in recent years owing to their unique radiation performance. Advantageous features include high directivities, high front-to-back ratios (FTBRs), wide beamwidths, and independence of large ground planes [1]-[8]. A variety of electrically small HDAs with specific characteristics have

Manuscript received September 20, 2020; revised December 18, 2020; and accepted December 19, 2020.

This work was supported in part by the National Natural Science Foundation of China contract number 62061006, in part by the Graduate Scientific Research and Innovation Foundation of Chongqing, China contract number CYB20066, in part by the Chongqing Natural Science Foundation contract number cstc2019jcyjjqX0004, and in part by the Australian Research Council grant number DP160102219.

M.-C. Tang, X. Chen, T. Shi, H. Tu, and Z. Wu are with the Key Laboratory of Dependable Service Computing in Cyber Physical Society Ministry of Education, School of Microelectronics and Communication Engineering, Chongqing University, Chongqing 400044, China (E-mail: tangmingchun@cqu.edu.cn)

R. W. Ziolkowski is with the University of Technology Sydney, Global Big Data Technologies Centre, Ultimo NSW 2007, Australia (E-mail: Richard.Ziolkowski@uts.edu.au). been developed that serve the needs of many specific spacelimited wireless communication systems. Single-polarization [1]-[4], dual-polarization [5], polarization reconfigurable [6], pattern reconfigurable [7], and non-Foster [8] versions have been designed and tested.

As fifth generation $(5 \mathrm{G})$ and beyond wireless ecosystems evolve, there is intense interest in compact, high directivity arrays with attractive performance characteristics to meet the demands of their stakeholders. Their applications include inband full-duplex (IBFD) and multiple-input-multiple-output (MIMO) operations. High density arrays of electrically small HDA elements would be very attractive candidates for those IBFD and MIMO assignments. This is particularly true if they were required for mobile, airborne or satellite platforms where space and weight constraints play defining roles.

IBFD antenna systems allow for transmitting and receiving signals with the same frequency at the same time [9], [10]. This technology can theoretically double system capacity, improve transfer data rates, and raise spectral efficiencies [11]. However, self-interference effects, i.e, the mutual couplings between the transmitter (Tx) and receiver ( $\mathrm{Rx}$ ) elements in the system, are a major hindrance to their high performance operation in current wireless systems [12]. Mutual coupling effects in high density arrays must be effectively suppressed to enhance their signal-to-interference-plus-noise ratio (SINR) features and, hence, their channel capacities, as well as to guarantee their signal quality and reliability for communication and remote sensing applications.

Many decoupling techniques/tools have been considered to reduce mutual coupling effects. These include, for example, hybrid meta-structures [13], mushroom electromagnetic band gap structures (EBG) [14], defected ground structures (DGS) [15], metamaterial polarization-rotator walls [16], angular offset elements [17], and parallel metal strips [18]. While effective, these approaches generally require the resulting centerto-center distances between the antenna elements to be larger than a half wavelength. This constraint hinders them from being employed in small-sized full-duplex antenna systems [13]-[17]. As an alternative, array-antenna decoupling surfaces (ADSs) [19], asymmetric loop resonators [20], isolation stubs [21], and waveguide metamaterials [22] have been shown to reduce mutual coupling when the element separation distance is much smaller than a half wavelength. Unfortunately, these decoupling technologies cannot be applied to realize the desired 
arrays of the noted HDA elements because large ground planes are required to achieve the reported radiation patterns and broadside gain values [18]-[22]. Another decoupling approach that does not require a large ground plane is the introduction of neutralization lines. However, they are usually applied to arrays of monopole antennas [23],[24] whose radiation behaviors cannot be guaranteed to be as directive as an HDA is, which limits their engineering applications.

Consequently, none of these many reported decoupling options would allow one to achieve a compact, high density array of HDAs for IBFD applications. Because of the requisite balance of their electric and magnetic elements and the lack of any ground plane, the strong coupling that occurs when they are placed in close proximity to each other leads to a deterioration of their combined radiation performance, i.e., their mutual couplings cause poor accepted power levels, distorted radiation patterns, degraded peak directivity values and decreased FTBR values. Thus, it is highly desirable to engineer an innovative decoupling method that would not only significantly reduce the mutual coupling between the HDA elements, but would also at least maintain the unidirectional radiation performance of each element. If a compact, lowprofile, high performance, unidirectional radiating array could be realized, it would significantly boost its applications in many space-limited wireless platforms.

A custom-designed decoupling structure is developed in this paper for integration with a compact HDA array to enable its application as an IBFD system. It is designed, simulated, fabricated and tested for operation around $f_{0}=1.511 \mathrm{GHz}$. The center-to-center distance between the HDA elements is only $0.3 \lambda_{0}$ at that center frequency. The specially engineered passive element is printed on a thin dielectric layer that lies directly on top of the array. The currents induced on it radiate scattered fields that effectively cancel the space wave couplings between the two radiating elements. The overall system is low profile with a total height of $\lambda_{0} / 20.3$. It is demonstrated that this passive scatterer is very effective and significantly reduces the mutual coupling between the HDA elements in this array. Moreover, it is verified that the Huygens source-based radiation performance characteristics for each HDA are maintained, i.e., their cardioid patterns, and the peak realized gain and FTBR values of the array witness improvements of $0.5 \mathrm{~dB}$ and $14.4 \mathrm{~dB}$, respectively.

All of the numerical simulations of the reported designs and their optimized configurations were performed using the commercial software ANSYS/Ansoft HFSS, version 18. These simulation models employed the known, real properties of all of the dielectrics and conductors employed in these systems.

\section{Two-ELEMENT HDA ARRAY WITH THE DECOUPLING STRUCTURE DESIGN}

The two-element HDA array configuration integrated with the decoupling structure is shown in Fig. 1. Its design parameters are also shown, and their optimized values are given in Table I. As shown in Fig. 1(a), the array consists of four circular substrate layers labeled as Layer_1, Layer_2, Layer_3, and Layer_4, respectively. All of these layers are Taconic TLY5 whose relative dielectric constant is $\varepsilon_{\mathrm{r}}=2.2$, loss tangent is $\tan \delta=0.0009$, and copper cladding thickness is $0.017 \mathrm{~mm}$. All of them have the same radius, $60 \mathrm{~mm}$. However, they have different thicknesses, i.e., $h_{1}=h_{2}=h_{3}=0.51 \mathrm{~mm}$, while $h_{4}=1.58 \mathrm{~mm}$. Four $3 \mathrm{D}$ printed nylon brackets $\left(\varepsilon_{\mathrm{r}}=\right.$ $\sim 2.4$ ) were employed to mechanically maintain the relative vertical distance between these layers. Our simulation results have shown that the $3 \mathrm{D}$ printed brackets have very little effect on the array's performance.

As shown in Figs. 1(a) and (b), each HDA consists of two NFRP elements, i.e., a magnetic element, which is a capacitively loaded loop (CLL); an electric element, which is an Egyptian axe dipole (EAD); and a coax-fed dipole antenna element. The operating frequency of the $50-\Omega$ source for each HDA is $f_{0}=1.51 \mathrm{GHz}$. All of the components of both HDAs have the same dimensions; the electrical size of each is $k a=0.76$ at $f_{0}$. The distance between the centers of the HDAs is set to $g_{1}=60 \mathrm{~mm}$, which corresponds to a center-to-center distance of $0.3 \lambda_{0}$, where $\lambda_{0}$ is the free-space wavelength corresponding to $f_{0}$. Thus, the total electrical size of the array is only $k a=1.81$.
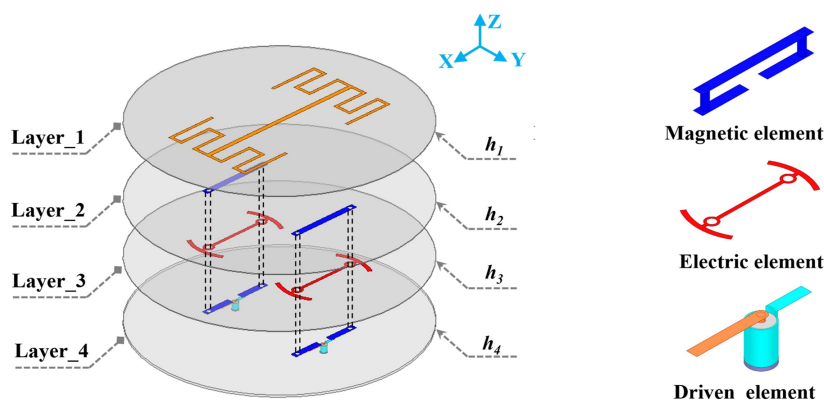

(a)

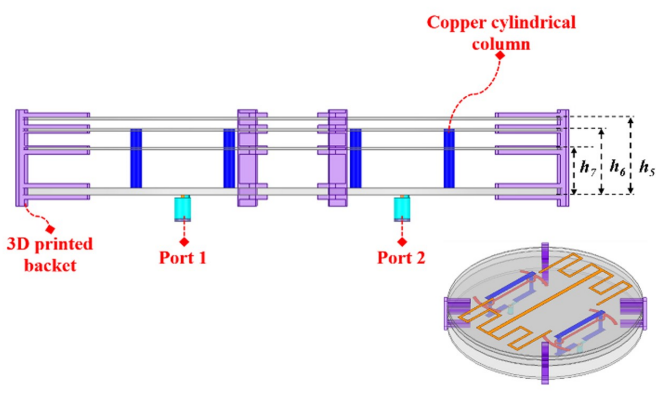

(b)

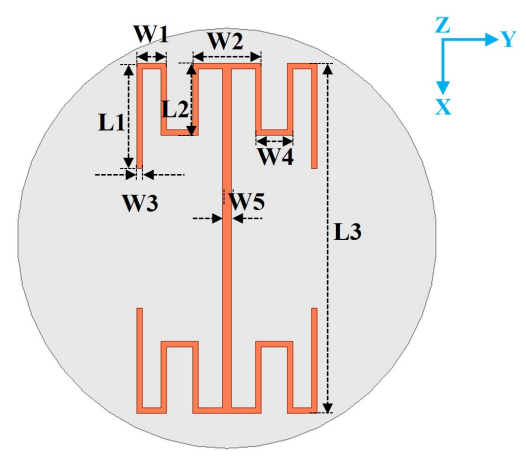

(c) 


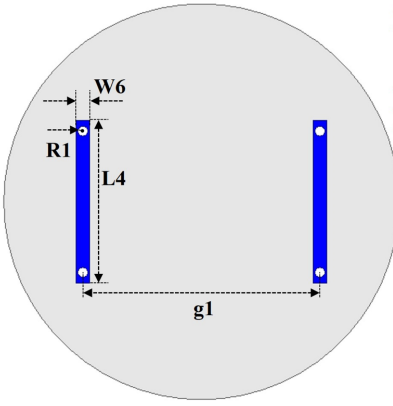

Upper surface of Layer_2

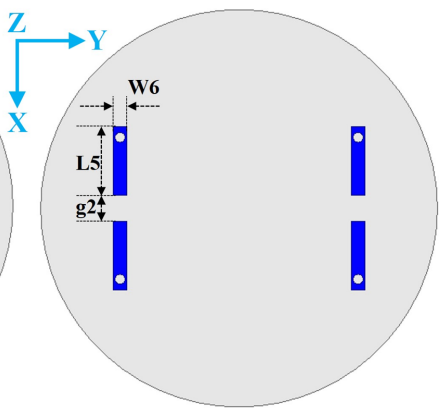

Upper surface of Layer_4

(d)

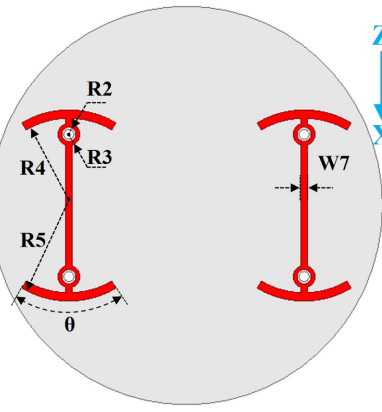

Upper surface of Layer_3

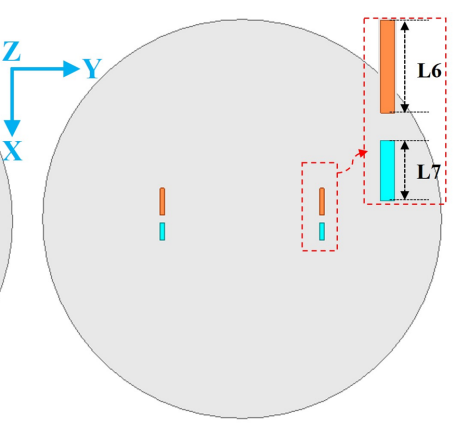

Bottom surface of Layer_4 (e)

Fig. 1. Configuration of the two-element HDA array integrated with the decoupling structure. (a) 3-D view. (b) Side view. (c) The decoupling structure on the upper surface of Layer_1. (d) Pieces of the CLL elements on the upper surfaces of Layer_2 and Layer_4. (e) The EAD elements on the upper surface of Layer_3 and the arms of the driven elements on the bottom surface of Layer_4.

Fig. 1(d) shows the top and bottom pieces of the CLL elements printed on Layer_2 and Layer_4. The single trace of each CLL element on Layer_2 is connected to its two traces on Layer_4 by two vertical, cylindrical copper columns as illustrated in Fig. 1(b). The gap of each CLL element on Layer_4 has a length $g_{2}=6.8 \mathrm{~mm}$. Since each CLL element is an LC resonator, this gap length helps adjust its resonance frequency. The two EAD elements are printed on the upper surface of Layer_3 as shown in Fig. 1(e). Four copper annular rings, each with the same inner radius, are introduced on the center traces of these EAD elements to avoid any direct connection to the four copper columns which penetrate through Layer_3. Fig. 1(e) indicates that the arms of the driven dipole are printed on the bottom of Layer_4. It is noted that the lengths of the two arms of each driven dipole are different. One is connected to the inner conductor of the coax probe; the other is connected to its outer conductor. This asymmetric configuration is different from the symmetric ones of the HDAs reported in [4] and [25]. It was found to provide more tuning freedom to further optimize the impedance matching and to facilitate improving the isolation level between the two ports of the HDA array.

In order to reduce the mutual coupling between the two elements, a pair of face-to-face oriented meander-line res-
TABLE I

Optimized Design Parameters OF THE Two-Element Huygens Dipole ANTENNA ARRAY AND ITS INTEgRATED DECOUPLING STRUCTURE

\begin{tabular}{|c|c|c|c|}
\hline$g_{1}=60 \mathrm{~mm}$ & $g_{2}=6.8 \mathrm{~mm}$ & $h_{1}=0.51 \mathrm{~mm}$ & $h_{2}=0.51 \mathrm{~mm}$ \\
\hline$h_{3}=0.51 \mathrm{~mm}$ & $h_{4}=1.58 \mathrm{~mm}$ & $h_{5}=9.76 \mathrm{~mm}$ & $h_{6}=8.45 \mathrm{~mm}$ \\
\hline$h_{7}=6.24 \mathrm{~mm}$ & $L_{1}=31 \mathrm{~mm}$ & $L_{2}=20.6 \mathrm{~mm}$ & $L_{3}=102 \mathrm{~mm}$ \\
\hline$L_{4}=41 \mathrm{~mm}$ & $L_{5}=17.1 \mathrm{~mm}$ & $L_{6}=10 \mathrm{~mm}$ & $L_{7}=6.5 \mathrm{~mm}$ \\
\hline$R_{1}=1.25 \mathrm{~mm}$ & $R_{2}=1.8 \mathrm{~mm}$ & $R_{3}=2.8 \mathrm{~mm}$ & $R_{4}=21.95 \mathrm{~mm}$ \\
\hline$R_{5}=24.05 \mathrm{~mm}$ & $W_{1}=8.6 \mathrm{~mm}$ & $W_{2}=17.6 \mathrm{~mm}$ & $W_{3}=1.6 \mathrm{~mm}$ \\
\hline$W_{4}=10.6 \mathrm{~mm}$ & $W_{5}=2.4 \mathrm{~mm}$ & $W_{6}=3.5 \mathrm{~mm}$ & $W_{7}=1.6 \mathrm{~mm}$ \\
\hline$\theta=60^{\circ}$ & & & \\
\hline
\end{tabular}

onators connected by a rectangular strip is printed on the upper surface of Layer_1, which lies on top of the HDA array, as shown in Figs. 1(a) and (c). The design of the decoupling element involved two main steps. The stages of its evolution in conjunction with the HDA array are depicted in Fig. 2. Array I, the first stage, represents the original HDA array without any decoupling element. Array II represents the second stage. The pair of face-to-face oriented meander-line resonators were placed initially on the top surface of Layer_2 and then on the top surface of the additional Layer_1 above the HDA array.
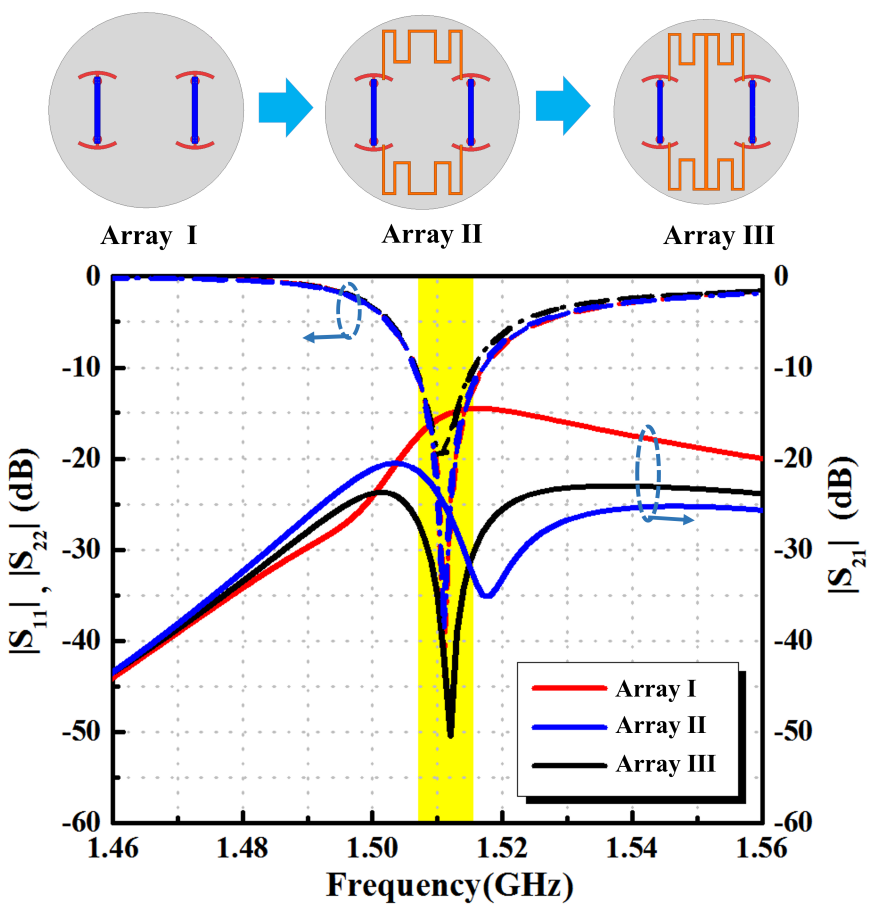

Fig. 2. The simulated $S$-parameters of the stages in the evolution of the twoelement HDA array with the decoupling element as functions of the source frequency. Array I: the original HDA array; Array II: the same HDA array loaded with a pair of a face-to-face oriented meander-line resonators; and Array III: the same HDA array loaded with the custom-designed decoupling element. The $-10-\mathrm{dB}$ impedance bandwidth is indicated by the yellow region.

The relative orientation of the resonators along both the $x$ and $y$-axis were considered, i.e., parallel and orthogonal to the $x$-axis orientations of the CLL and EAD elements. Numerical parameter studies were performed to tune the performance of each configuration. It was found that the additional layer, 
orthogonal orientation arrangement, was superior, and the optimized resonators achieved a reduction of the mutual coupling level between the two ports by about $12.5 \mathrm{~dB}$. Array III represents the third and final stage. The rectangular strip was introduced to connect the centers of the two meanderline resonators together. It was hoped that it would impact the $x$-directed components of the electric fields radiated by the currents on the tops of the CLL elements. Another suite of parameter studies was performed to realize the optimized design. This symmetric structure further improved the isolation level between the two ports by about $7.5 \mathrm{~dB}$.

There exist strong space waves that couple the two HDAs when the array has no decoupling structure. They cause poor isolation between the two ports. As will be explained below, the fields radiated by both HDAs induce currents on this decoupling element. The scattered fields that it re-emits when its dimensions are optimized cancel the space waves that couple the two HDAs, producing a very high isolation of their two ports.

Fig. 2 shows the simulated S-parameter values as functions of the source frequency for the entire system without any decoupling element, i.e., Array I. As the plots of $\left|S_{11}\right|$ and $\left|S_{22}\right|$ indicate, the resonance frequency of each port is centered at $1.512 \mathrm{GHz}$ with $\left|S_{11}\right|_{\min }=\left|S_{22}\right|_{\min }=-42 \mathrm{~dB}$. The impedance bandwidth, where $\left|S_{11}\right|_{\min }$ and $\left|S_{22}\right|_{\min } \leq-10 \mathrm{~dB}$, for both HDAs is $10.7 \mathrm{MHz}$. However, the mutual coupling between the two ports is high with $\left|S_{21}\right|=\left|S_{12}\right|$ reaching approximately $-14.4 \mathrm{~dB}$ within that operational bandwidth.

Fig. 2 also shows the corresponding simulated $S$-parameter values for Array III. As the plots of $\left|S_{11}\right|$ and $\left|S_{22}\right|$ indicate, the resonance frequency of each port is now centered at 1.511 GHz with $\left|S_{11}\right|_{\text {min }}=-20 \mathrm{~dB}$ and $\left|S_{22}\right|_{\min }=-19 \mathrm{~dB}$. Comparing the two cases, the frequency shift is a mere $0.066 \%$. The corresponding impedance bandwidth of each HDA, $9.2 \mathrm{MHz}$, is a slightly smaller. On the other hand, the isolation between the two ports when the decoupling element is present is considerably higher textcolorred with its maximum level at $50.4 \mathrm{~dB}$ around $f_{0}$, i.e, $\left|S_{21}\right|$ is less than $-50 \mathrm{~dB}$ within the operational bandwidth.

Fig. 3 shows the simulated realized gain patterns of Array I and Array III. They clearly demonstrate the impact of the mutual coupling when the decoupling element is not present and the significant impact it has on the radiation performance when it is, i.e., when the mutual coupling is effectively absent. When only one port is excited and the other is terminated with a $50-\Omega$ load, as indicated in Figs. 3(a) and (b), the realized gain patterns of each element in either of these states are degraded because of strong mutual coupling. This degradation is particularly noticeable in the $H$-plane. The peak of the realized gain pattern deviates significantly from the $+z$ axis by approximately $30^{\circ}$. Moreover, with only one HDA element active, the induced currents on the two meanderlines are larger than those on the middle rectangular strip of the decoupling structure. This behavior results in the increased cross polarization levels observed in Figs. 3(a) and (b).

On the other hand, the broadside radiation patterns are well recovered after the HDA array is loaded with the decoupling element. Moreover, there is an improvement in both the peak

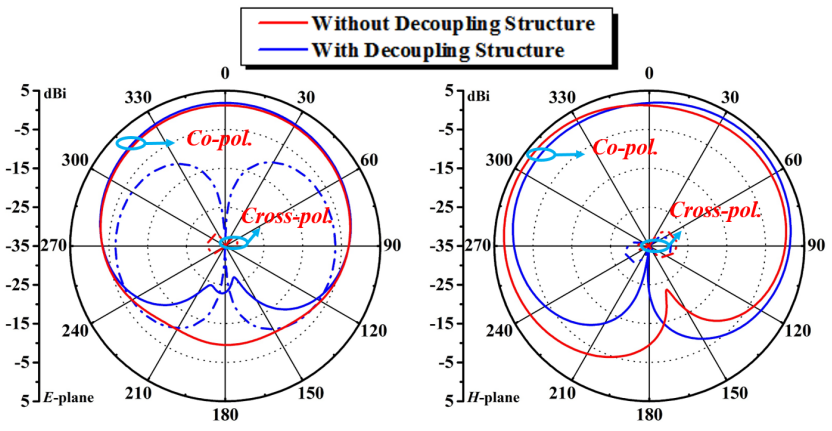

(a)

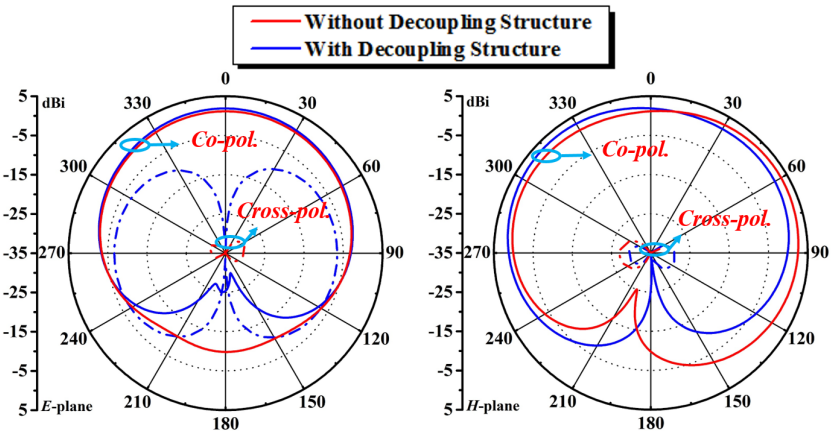

(b)

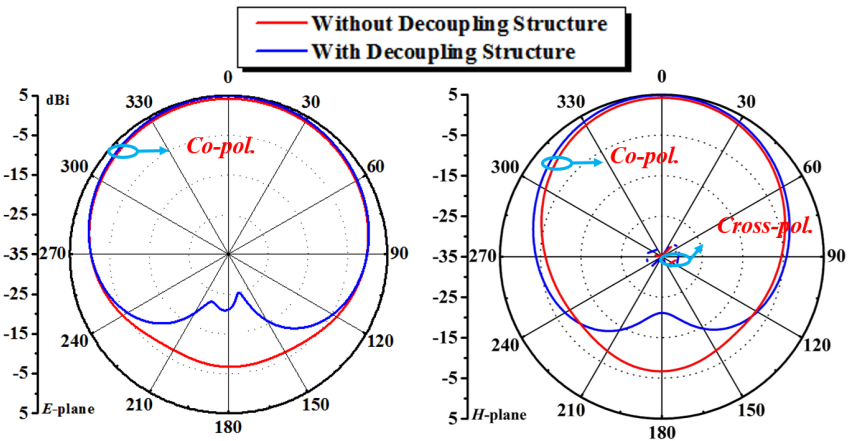

(c)

Fig. 3. Comparisons of the simulated realized gain patterns in the $E(z 0 x)$ and $H(z 0 y)$ planes for the HDA array with and without decoupling structure at its corresponding resonant frequency, i.e., at $1.511 \mathrm{GHz}$ without the decoupling structure and at $1.512 \mathrm{GHz}$ with it. (a) Port 1 is excited, while Port 2 is terminated with a $50-\Omega$ load. (b) Port 2 is excited, while Port 1 is terminated with a $50-\Omega$ load. (c) Both ports are excited in-phase.

realized gain and FTBR values, as well as the shape of the pattern in the $H$-plane. Notice that in both states when the decoupling element is present, the half-power beamwidths (HPBWs) in the $E$-plane and the $H$-plane are approximately $155^{\circ}$ and $230^{\circ}$, respectively. These values are much greater than the HPBW, $131^{\circ}$, of a typical HDA alone [25].

Finally, the simulated realized gain patterns for both arrays when both of their ports are excited is shown in Fig. 3(c). The presence of decoupling structure improves the broadside peak realized gain value to $4.88 \mathrm{dBi}$, an increase of $0.5 \mathrm{~dB}$. It also significantly increases the corresponding FTBR value by 14.4 to $25.5 \mathrm{~dB}$. The cross-polarization levels in the E-plane are now below the $-35 \mathrm{~dB}$ threshold of the plot and very close to it in the H-plane. 


\section{The Decoupling Structure's Operational MECHANISMS}

To understand how the decoupling structure operates, a comprehensive investigation of the currents induced on it and the resulting scattered fields emitted by it was undertaken. Its operational mechanisms will be described with the analysis of two fundamental states of the system. Only one HDA element is active and the other is passive and matched to a $50-\Omega$ load in the first case. Both HDAs are active in the second case.

\section{A. Case I: Only One HDA Is Active}

Fig. 4(a) presents the simulated surface current distributions on the top trace of both CLL elements and the decoupling structure when only the HDA element on the $-y$ side of the origin is active. The main directions of those currents are emphasized with the red and blue arrows, respectively. Because the induced current on the passive HDA is quite small, there is no red arrow present. Note that the current distributions on the decoupling structure do not behave as a single classical electric dipole along the $x$-axis. Instead, there are strong induced currents on its two meander-line resonators along the $y$-axis and a much weaker current on its center trace along the $x$-axis. The currents on the meander-line segments of the decoupling element are in opposite directions. The current on its middle trace is oriented opposite to that of the current on the upper trace of the CLL element of the active HDA. Consequently, fields radiated by the array when only one HDA active consist of a combination of those radiated by the pair of electric and magnetic NFRP elements of the active HDA and three electrically small electric dipoles associated with the decoupling element. This arrangement is illustrated in Fig. 4(b).

An analytic expression for the total electric field radiated into the far field is obtained in this case as follows. Since all of the elements are electrically small, constant electric and magnetic current densities are associated with the appropriate elements, i.e., electric (magnetic) ones are associated with the E-labeled (M-labeled) arrows in Fig. 4(b). Consider continuous wave $(\mathrm{CW})$ electric and magnetic elemental current densities $\vec{J}$ and $\vec{K}$ at the angular frequency $\omega=2 \pi f$ that are located at the same point $r_{0}=x_{0} \hat{x}+y_{0} \hat{y}$ in the $x y$-plane and that are oriented in the $\hat{u}$ and $\hat{v}$ directions, respectively. They are represented as:

$$
\begin{array}{r}
\vec{J}=I_{e} \ell_{e} \delta\left(x-x_{0}\right) \delta\left(y-y_{0}\right) \delta(z) \hat{u} \\
\vec{K}=I_{m} \ell_{m} \delta\left(x-x_{0}\right) \delta\left(y-y_{0}\right) \delta(z) \hat{v}
\end{array}
$$

where $I_{e} \ell_{e}$ and $I_{m} \ell_{m}$ represent the associated electric and magnetic current moments, respectively. The electric fields that these $x y$-plane sources radiate into the far field referred to the coordinate origin are given by the expressions [26]:

$$
\begin{aligned}
& \vec{E}_{\omega, J}^{f f}(\vec{r})=+j \omega \mu I_{e} \ell_{e} G(r) e^{+j k \hat{r} \cdot \vec{r}_{0}}(\hat{r} \times \hat{r} \times \hat{u}) \\
& \vec{E}_{\omega, K}^{f f}(\vec{r})=+j \omega \mu\left(\frac{I_{m} \ell_{m}}{\eta}\right) G(r) e^{+j k \hat{r} \cdot \vec{r}_{0}}(\hat{r} \times \hat{v})
\end{aligned}
$$

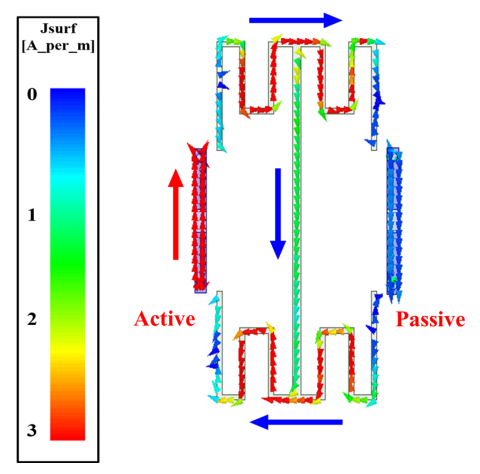

(a)

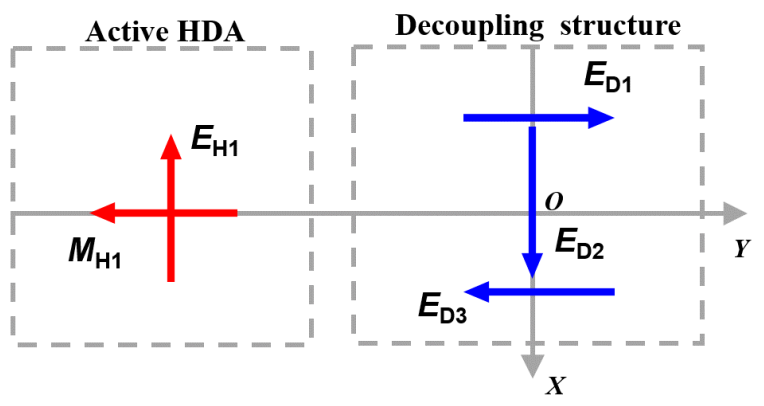

(b)

Fig. 4. The HDA array augmented with the decoupling element when only one HDA is active. (a) The current distributions on the decoupling element and the top traces of the CLL elements of both HDAs. (b) The equivalent set of the main electric and magnetic radiating elements.

where $\mu, k$, and $\eta$ are the free-space permeability, wave number and wave impedance, respectively. The corresponding Greens function of the free-space Helmholtz equation is

$$
G(r)=\frac{e^{-j k r}}{4 \pi r}
$$

The elemental radiators in the model illustrated in Fig. 4(b) combine to form an elemental HDA, a two-element electric Hertzian dipole (EHD) array, and a single EHD. The elemental HDA consists of a balanced combination of an electric and a magnetic dipole, the balanced condition being $I_{e} \ell_{e}=I_{m} \ell_{m} / \eta \equiv I_{0} \ell_{0}$ [25]. Let $d_{y} / 2$ represent the distance of either HDA from the origin along the $y$-axis. Physically, $d_{y}=0.3 \lambda_{0}$. The far-field electric field of the active HDA is obtained from (2) as

$$
\begin{aligned}
E_{\omega, \mathrm{HDA}}^{f f}(\vec{r}) & =+j \omega \mu I_{0} \ell_{0} G(r) \\
& \times e^{-j\left(k d_{y} / 2\right) \sin \theta \sin \phi}{\overrightarrow{\operatorname{EVF}_{\mathrm{HDA}}}}(\theta, \phi)
\end{aligned}
$$

where the HDA's element vector factor

$$
\overrightarrow{\operatorname{EVF}}_{\mathrm{HDA}}(\theta, \phi)=-(\hat{r} \times \hat{r} \times \hat{x}+\hat{r} \times \hat{y})
$$

Similarly, let $d_{x} / 2$ be the distance from the origin along the $x$-axis of both of the $y$-oriented electric dipoles corresponding 
to the meander-line resonators. Physically, $d_{x}=0.51 \lambda_{0}$. The associated electric current moments are $I_{D 3} \ell_{D 3}=-I_{D 1} \ell_{D 1}$. Consequently, the far-field electric field of this two-element EHD array is

$$
E_{\omega, \mathrm{DA}}^{f f}(\vec{r})=+j \omega \mu G(r) \mathrm{AF}_{\mathrm{DA}}(\theta, \phi) \overrightarrow{\mathrm{EVF}}_{\mathrm{DA}}(\theta, \phi)
$$

where its array factor is

$$
\begin{aligned}
\operatorname{AF}_{\mathrm{DA}}(\theta, \phi)= & I_{D 1} \ell_{D 1} e^{-j\left(k d_{x} / 2\right) \sin \theta \cos \phi} \\
& +I_{D 3} \ell_{D 3} e^{+j\left(k d_{x} / 2\right) \sin \theta \cos \phi} \\
= & -2 j I_{D 1} \ell_{D 1} \sin \left[\left(\frac{k d_{x}}{2}\right) \sin \theta \cos \phi\right]
\end{aligned}
$$

and its element vector factor is

$$
\overrightarrow{\operatorname{EVF}}_{\mathrm{DA}}(\theta, \phi)=\hat{r} \times \hat{r} \times \hat{y}
$$

Finally, let the electric dipole of the center-line of the decoupling element be centered at the origin. Let its electric current moment be $I_{D 2} \ell_{D 2}$. Thus, the far-field electric field is simply:

$$
E_{\omega, \mathrm{D}}^{f f}(\vec{r})=+j \omega \mu I_{D 2} \ell_{D 2} G(r)(\hat{r} \times \hat{r} \times \hat{x})
$$

Writing the radial unit vector in terms of cartesian coordinates, $\hat{r}=\sin \theta \cos \phi+\sin \theta \sin \phi+\cos \theta$, simplifies understanding the contributions from the various radiating elements whose current moments are defined relative to them.

$$
\begin{aligned}
\hat{r} \times \hat{x}= & \cos \theta \hat{y}-\sin \theta \sin \phi \hat{z} \\
\hat{r} \times \hat{r} \times \hat{x}= & -\left(\sin ^{2} \theta \sin ^{2} \phi+\cos ^{2} \theta\right) \hat{x} \\
& +\sin \theta \sin \phi \cos \phi \hat{y}+\sin \theta \cos \theta \cos \phi \hat{z} \\
\hat{r} \times \hat{y}= & -\cos \theta \hat{x}+\sin \theta \cos \phi \hat{z} \\
\hat{r} \times \hat{r} \times \hat{y}= & \sin ^{2} \theta \sin \phi \cos \phi \hat{x} \\
- & \left(\sin ^{2} \theta \cos ^{2} \phi+\cos ^{2} \theta\right) \hat{y}+\sin \theta \cos \theta \sin \phi \hat{z}
\end{aligned}
$$

The total electric field radiated into the far-field in this Case I model:

$$
E_{\omega, \text { total }}^{f f}(\vec{r})=E_{\omega, \mathrm{HDA}}^{f f}(\vec{r})+E_{\omega, \mathrm{DA}}^{f f}(\vec{r})+E_{\omega, \mathrm{D}}^{f f}(\vec{r})
$$

is obtained by combining (4), (6), and (9). One can then calculate the far-field total Poyntings vector as:

$$
\mathrm{S}_{\omega, \text { total }}^{f f}(\vec{r})=\frac{1}{2 \eta}\left|\vec{E}_{\omega, \text { total }}^{f f}(\vec{r})\right|^{2}
$$

the total power radiated (the total flux of the Poyntings vector through the sphere at infinity $S_{\infty}^{2}$ ) as:

$$
\mathrm{P}_{\text {total }}^{r a d}=\oiint_{S_{\infty}^{2}} d \Omega\left[\hat{r} \cdot \mathrm{S}_{\text {total }}^{f f}(\vec{r})\right]
$$

where $d \Omega=r^{2} \sin \theta d \theta d \phi$, and finally the directivity as

$$
D_{\text {total }}(\theta, \phi)=\lim _{r \rightarrow \infty} \frac{4 \pi r^{2}\left[\hat{r} \cdot \mathrm{S}_{\text {total }}^{f f}(\vec{r})\right]}{P_{\text {total }}^{r a d}}
$$

are obtained straightforwardly from (12). A MATLAB program was constructed from these expressions to calculate the corresponding directivity patterns.

Fig. 5 compares the analytical Case I model's directivity patterns with the corresponding simulated ones. The theoretical radiation patterns were obtained with the magnitudes of the three electric dipoles satisfy the ratio as $I_{0} \ell_{0}: I_{D 1} \ell_{D 1}$ : $I_{D 2} \ell_{D 2}: I_{D 3} \ell_{D 3}=1: 0.6: 0.12:-0.6$. These values were obtained by carefully comparing the analytical results with the simulated ones. Note that the simulated $H$-plane radiation patterns witness a slight tilt from the broadside direction. More simulations confirmed that it arises from the small currents induced on the passive HDA that were neglected in constructing the Case I model shown in Fig. 4(b). Overall, the shapes of the analytical results are in very good agreement with the simulated ones.

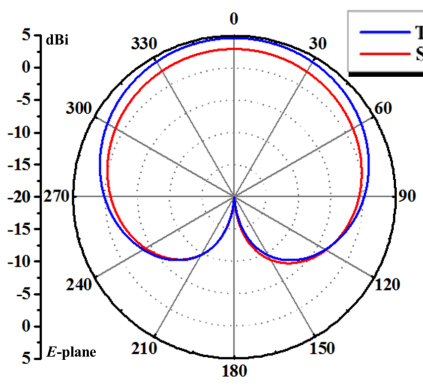

(a)

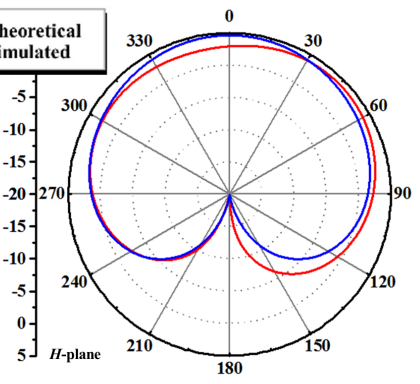

(b)
Fig. 5. The theoretical and simulated directivity patterns of the HDA array when only one HDA is active. (a) $E$-plane. (b) $H$-plane.

Specific details of both the analytical model and the simulated results for this Case I are summarized in Table II. They too are in very reasonable agreement even with the simplicity of the theoretical model. Since the ratio $I_{0} \ell_{0}: I_{D 1} \ell_{D 1}$ : $I_{D 2} \ell_{D 2}: I_{D 3} \ell_{D 3}$ is important to optimize the isolation level and maintain the broadside radiation behavior of each element, the magnitudes of $I_{D 1} \ell_{D 1}, I_{D 2} \ell_{D 2}$, and $I_{D 3} \ell_{D 3}$ are effectively optimized by simply adjusting the capacitive coupling between the decoupling element and the HDA array. The strength of the coupling is modified simply by changing the height between the decoupling element and the upper surface of Layer_2 in Fig. 1. The optimal value of the height was obtained straightforwardly by means of several simulations.

\section{B. Case II: Both HDAs Active}

Now consider the two-element array with both of its HDAs being active. Fig. 6(a) presents the simulated surface current distributions on the top traces of both CLL elements and the decoupling structure. The main current directions are indicated with the red (HDAs) and blue (decoupling element) arrows. Since both HDAs are excited in-phase with the same 
TABLE II

COMPARISON OF THE THEORETICAL AND SiMUlation RESUltS FOR THE Two-Element Huygens Dipole Array With the Decoupling StRucture When ONLY ONE HDA Is ACTIVE

\begin{tabular}{|c|c|c|}
\hline & $\begin{array}{c}\text { Theoretical } \\
\text { Results }\end{array}$ & $\begin{array}{c}\text { Simulated } \\
\text { Results }\end{array}$ \\
\hline Peak directivity & $4.61 \mathrm{dBi}$ & $4.08 \mathrm{dBi}$ \\
\hline Peak realized gain & - & $3.10 \mathrm{dBi}$ \\
\hline HBPW in $E$-plane & $\sim 148^{\circ}$ & $\sim 155^{\circ}$ \\
\hline HPBW in $H$-plane & $\sim 174^{\circ}$ & $\sim 230^{\circ}$ \\
\hline FTBR & $24.9 \mathrm{~dB}$ & $23.0 \mathrm{~dB}$ \\
\hline Radiation efficiency & - & $80.1 \%$ \\
\hline
\end{tabular}

magnitudes and the decoupling structure is symmetric about the $x$-axis, the current distributions on their CLL elements are the same and large. In contrast to those in Fig. 4(a), the induced currents on each of the two meander-line ends of the decoupling element now have opposite orientations. They contribute to a lower cross polarization level in comparison to that of Case I. As indicated in Fig. 6(b), the equivalent analytic model must now accommodate the electric and magnetic dipoles of each HDA and the 5 electric dipoles associated with the decoupling element.

Extending the analysis presented above, the two HDAs radiate the same electric field into the far field with weighted by phase term associated with their displacement from the origin:

$$
E_{\omega, \mathrm{HDAs}}^{f f}(\vec{r})=+j \omega \mu G(r) \operatorname{AF}_{\mathrm{HDAs}}(\theta, \phi) \overrightarrow{\mathrm{EVF}}_{\mathrm{HDA}}(\theta, \phi)
$$

where the array factor is

$$
\begin{aligned}
& \operatorname{AF}_{\mathrm{HDAs}}(\theta, \phi)=I_{0} \ell_{0} {\left[e^{-j\left(k d_{y} / 2\right) \sin \theta \sin \phi}\right.} \\
&\left.+e^{+j\left(k d_{y} / 2\right) \sin \theta \sin \phi}\right] \\
&=2 I_{0} \ell_{0} \cos \left[\left(\frac{k d_{y}}{2}\right) \sin \theta \sin \phi\right]
\end{aligned}
$$

The centers of the meander-line dipoles are now offset from the $y$-axis. Let this offset distance be labeled $\delta_{y}$. One can easily deduce from Fig. 6(a) that physically $\delta_{y}=d_{y} / 2$ in this model. Then the source vectors of these dipoles are summarized as: $\vec{r}_{0}= \pm\left(d_{x} / 2\right) \hat{x} \pm\left(d_{y} / 2\right) \hat{y}$. The electric current moments of these four dipoles are labeled: $I_{D 1} \ell_{D 1}$, $I_{D 3} \ell_{D 3}, I_{D 4} \ell_{D 4}$, and $I_{D 5} \ell_{D 5}$. With the indicated directions in Fig. 6(b) and the same magnitudes of the current densities on the top and bottom meander-line segments, these current moments become: $-I_{D 3} \ell_{D 3}=+I_{D 1} \ell_{D 1} \equiv I_{1} \ell_{1}$ and $-I_{D 4} \ell_{D 4}=+I_{D 5} \ell_{D 5} \equiv I_{1} \ell_{1}$. These four dipoles form an array whose electric field in the far field is

$$
E_{\omega, 4 \mathrm{Ds}}^{f f}(\vec{r})=+j \omega \mu G(r) \mathrm{AF}_{4 \mathrm{Ds}}(\theta, \phi){\overrightarrow{\mathrm{EVF}_{\mathrm{DA}}}}_{(\theta, \phi)}
$$

where the array factor is now

$$
\begin{aligned}
\operatorname{AF}_{4 \mathrm{Ds}}(\theta, \phi) & =I_{D 1} \ell_{D 1} e^{-j\left(k \delta_{y} / 2\right) \sin \theta \sin \phi} e^{-j\left(k d_{x} / 2\right) \sin \theta \cos \phi} \\
& +I_{D 3} \ell_{D 3} e^{+j\left(k \delta_{y} / 2\right) \sin \theta \sin \phi} e^{-j\left(k d_{x} / 2\right) \sin \theta \cos \phi} \\
& +I_{D 4} \ell_{D 4} e^{-j\left(k \delta_{y} / 2\right) \sin \theta \sin \phi} e^{+j\left(k d_{x} / 2\right) \sin \theta \cos \phi} \\
& +I_{D 5} \ell_{D 5} e^{+j\left(k \delta_{y} / 2\right) \sin \theta \sin \phi} e^{+j\left(k d_{x} / 2\right) \sin \theta \cos \phi} \\
& =-4 I_{1} \ell_{1}\left\{\sin \left[\frac{k d_{x}}{2} \sin \theta \cos \phi\right]\right. \\
& \left.\times \sin \left[\frac{k \delta_{y}}{2} \sin \theta \sin \phi\right]\right\}
\end{aligned}
$$

and the element vector factor is the same as (8). Finally, the far-field electric field corresponding to the center trace of the decoupling element remains as (9).
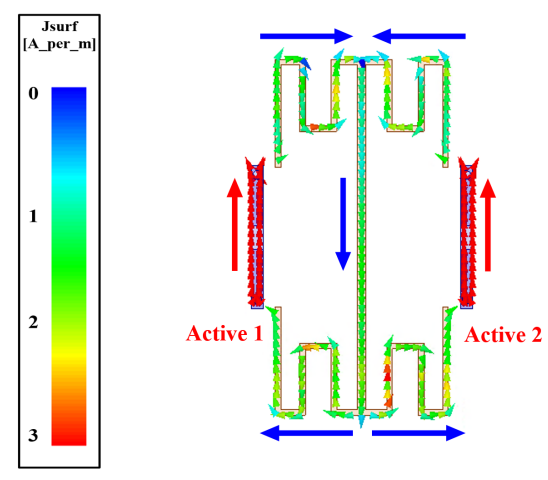

(a)

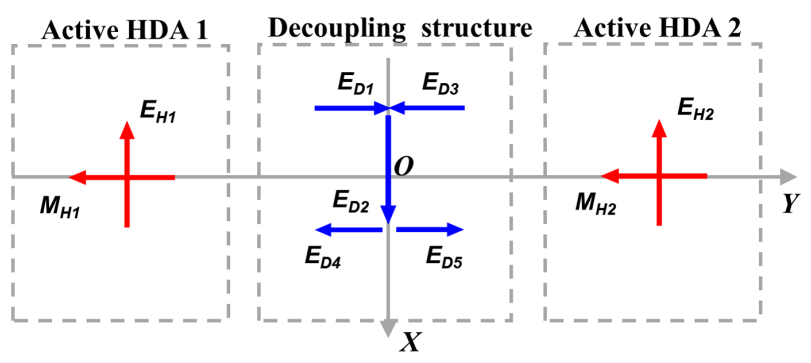

(b)

Fig. 6. The HDA array with decoupling structure when both HDAs are active. (a) The current distributions on the decoupling structure and the top traces of the CLL elements. (b) The equivalent model of electric and magnetic dipoles.

The analytical total electric field in the far field when both HDAs are active is:

$$
E_{\omega, \text { total }}^{f f}(\vec{r})=E_{\omega, \mathrm{HDAs}}^{f f}(\vec{r})+E_{\omega, 4 \mathrm{Ds}}^{f f}(\vec{r})+E_{\omega, \mathrm{D}}^{f f}(\vec{r})
$$

The analytical directivity patterns obtained with this expression and the corresponding simulated ones are compared in Fig. 7. The current moment ratios of the theoretical model were determined to be $I_{0} \ell_{0}: I_{1} \ell_{1}: I_{D 2} \ell_{D 2}=1.0: 0.5: 0.2$. The theoretical directivity patterns in both principal planes are in quite nice agreement with their simulated ones. Specific details of both the analytical model and the simulated results for this Case II are summarized in Table III. They too are in very reasonable agreement even with the simplicity of the theoretical model. Compared with the directivity value in Case 
I, its value in Case II is $5.71 \mathrm{dBi}$, a $1.16 \mathrm{~dB}$ increase. This value is close to the ideal increase without considering the coupling effect, $1.63 \mathrm{~dB}$ [35], [36]. It is noted that the two HDA combination does not enhance the directivity by $3 \mathrm{~dB}$. This outcome is a result of the $0.3 \lambda_{0}$ distance of separation between those elements. The directivity of the two-element HDA array alone with the element separation being $0.3,0.4$, 0.5 , and $0.6 \lambda_{0}$ is, respectively, 5.87, 6.64, 7.45, and $8.14 \mathrm{dBi}$. The anticipated $3 \mathrm{~dB}$ enhancement is approximately obtained with the largest element separation distance. With the elements being quite close to each other, the full interference effects of their fields are not well expressed. At yet further separation distances, the interference patterns begin to degrade the overall performance. The smallest separation distance was of most interest since it greatly challenges the design of the decoupling element and because it facilitates the realization of a practical compact, dense array for IBFD applications with an effective decoupling element.

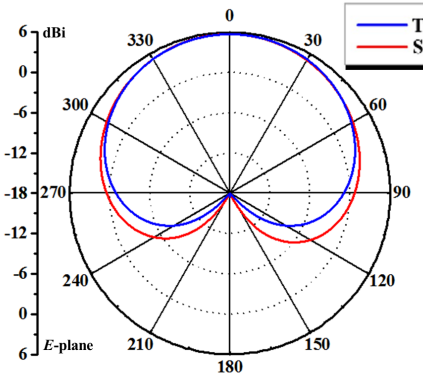

(a)

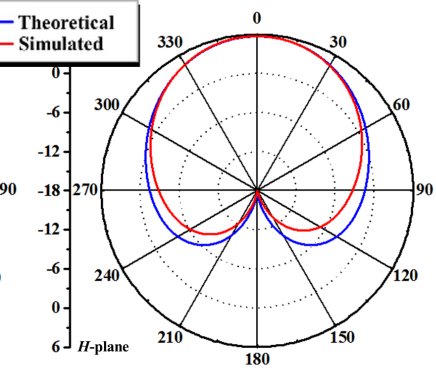

(b)
Fig. 7. The theoretical and simulated directivity patterns of the HDA array when both HDAs are active. (a) $E$-plane. (b) $H$-plane.

TABLE III

COMPARISON OF THE THEORETICAL AND Simulation RESUlts FOR THE Two-Element Huygens Dipole Array With the Decoupling Structure When Both HDAs Are Active

\begin{tabular}{|c|c|c|}
\hline & $\begin{array}{c}\text { Theoretical } \\
\text { Results }\end{array}$ & $\begin{array}{c}\text { Simulated } \\
\text { Results }\end{array}$ \\
\hline Peak directivity & $5.69 \mathrm{dBi}$ & $5.71 \mathrm{dBi}$ \\
\hline Peak realized gain & - & $4.88 \mathrm{dBi}$ \\
\hline HPBW in $E$-plane & $\sim 128^{\circ}$ & $\sim 135^{\circ}$ \\
\hline HPBW in $H$-plane & $\sim 94^{\circ}$ & $\sim 87^{\circ}$ \\
\hline FTBR & $23.09 \mathrm{~dB}$ & $25.5 \mathrm{~dB}$ \\
\hline Radiation efficiency & - & $83 \%$ \\
\hline
\end{tabular}

\section{Simulated and Measured Results}

The HDA array with the integrated decoupling element was fabricated, assembled, and tested. Photos of its component parts and the assembled prototype are presented in Figs. 8(a) and (b), respectively. Two sleeve baluns with a length of $43 \mathrm{~mm}\left(\sim 0.21 \lambda_{0}\right)$ were employed, one for each port, to eliminate the induced leakage currents on the outer walls of the long cables in the measurements [27]. Their presence ensured a proper comparison with the simulated performance characteristics. The baluns and their inclusion in the measured prototype are also shown in Figs. 8(a) and 8(b), respectively. The $S$-parameters of the prototype were measured with an Agilent E8361A PNA vector network analyzer (VNA). The far-field radiation performance characteristics were measured in an anechoic chamber at the University of Electronic Science and Technology of China (UESTC), Chengdu, China. The antenna under test (AUT) in this chamber is shown in Fig. 8(c).

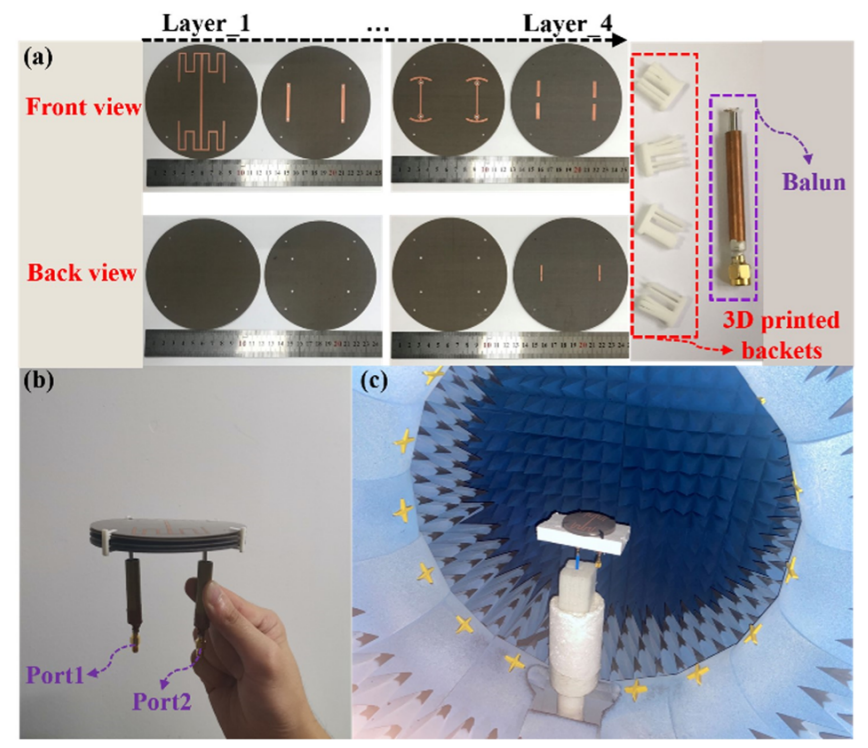

Fig. 8. Fabricated prototype of the HDA array with its custom-designed decoupling element. (a) Front and back views of each layer before assembly. (b) Side view of the assembled array mounted on two sleeve baluns, one for each port. (c) 3-D isometric view of the AUT in the anechoic measurement chamber.

The measured and simulated performance characteristics of the HDA array are presented in Figs. 9 and 10. As shown in Fig. 9, the measured (simulated) -10-dB overlapping impedance bandwidth, i.e., the frequencies for which $\left|S_{11}\right|_{\text {min }}$ $\leq-10 \mathrm{~dB}$ for both ports was $8.0 \mathrm{MHz}$, covering from the range from 1.507 to $1.515 \mathrm{GHz}(10 \mathrm{MHz}, 1.506$ to $1.516 \mathrm{GHz})$. The measured (simulated) center-to-center physical distance between the two elements at the center of the operational frequency range $f_{0}, 1.511 \mathrm{GHz}(1.511 \mathrm{GHz})$ was $d_{y}=0.302 \lambda_{0}$ ( $\left.0.301 \lambda_{0}\right)$. The corresponding fractional impedance bandwidth (FBW) was $0.53 \%(0.66 \%)$.

The measured (simulated) peak isolation level between the two ports was greater than $58(50.4) \mathrm{dB}$, which is much higher than the standard requirement for most high-density phased antenna arrays. In fact, the isolation level in practical applications is commonly expected to be only higher than 30 $\mathrm{dB}$ for an inter-element distance of $0.5 \lambda_{0}$ [13]. The average isolation level of the prototype HDA array is $\sim 35 \mathrm{~dB}$ for the inter-element distance being only $0.3 \lambda_{0}$. This result is deemed reasonably high enough [34], [35] and is demonstrated below to be comparable to recently reported narrowband IBFD antennas. It is important to note that the average isolation 
level between the two ports of this prototype is much higher than that attained with the previously reported dual linearly polarized (dual-LP) HDA (25.8 dB) [5], which achieved that isolation level because of its orthogonal polarization states.

Moreover, the polarization of the fields radiated by both ports of the developed prototype are more uniform with lower cross-pol. levels, i.e., the polarization purity performance is better. This is yet another of its advantages since it is wellknown that IBFD systems which have high quality, uniform polarization performance from both ports are especially desirable in many applications [28], [33].

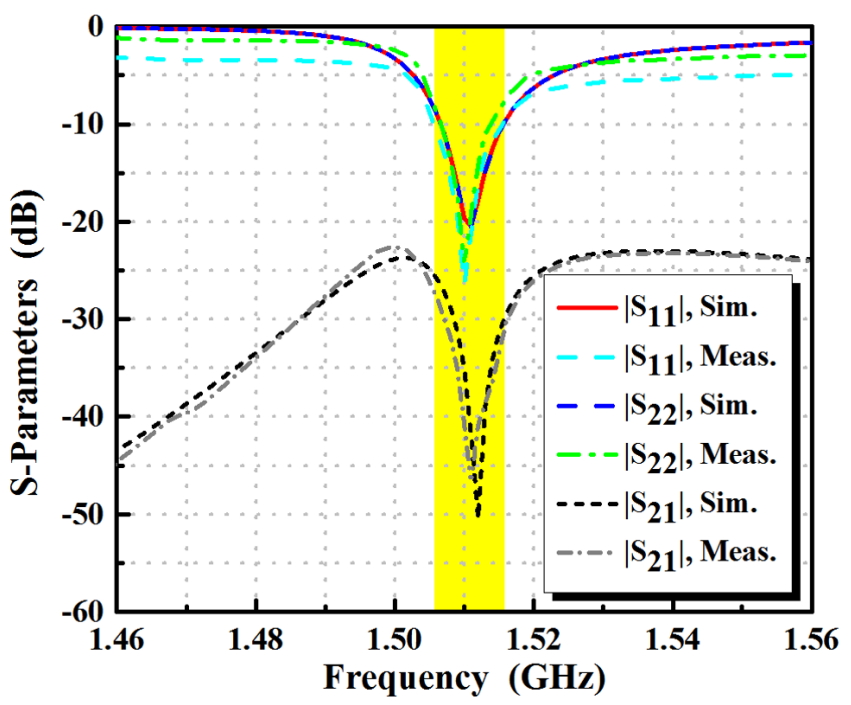

Fig. 9. Measured and simulated S-parameters of the HDA array with its custom-designed decoupling element. The $-10-\mathrm{dB}$ impedance bandwidth is indicated by the yellow region.

The measured and simulated realized gain patterns in the $E$ - and $H$ - planes are compared in Fig. 10. The measured (simulated) peak realized gain value is $2.58(3.10) \mathrm{dBi}$ at the resonance frequency $1.511(1.511) \mathrm{GHz}$ with the FTBR $=23.4$ (23.0) $\mathrm{dB}$ and the radiation efficiency $(\mathrm{RE})=76 \%(80.1 \%)$. The measured (simulated) half-power beamwidths were $169^{\circ}$, from $-83^{\circ}$ to $86^{\circ}\left(179^{\circ}\right.$, from $-89^{\circ}$ to $\left.90^{\circ}\right)$ in the $z 0 x$-plane and $214^{\circ}$, from $-103^{\circ}$ to $111^{\circ}\left(223^{\circ}\right.$, from $-119^{\circ}$ to $\left.104^{\circ}\right)$ in the $z 0 y$-plane, respectively. Note that the half-power beamwidth in the $H$-plane is much larger than that in $E$-plane because the two HDAs are separated along the $y$-axis. The value is larger than the theoretical half-power beamwidth of a standard electrically small Huygens dipole antenna, $131^{\circ}$ [25], which further confirms the design principles described in Section III. Notice that the cross-polarization levels are very small; the decoupling element also helps maintain the polarization purity of each HDA.

A performance comparison of the prototype with recently reported narrow bandwidth antennas applied in IBFD systems, e.g., with fractional bandwidths $<6 \%$, are given in Table V. Those previously reported systems all utilized polarization diversity to decrease the isolation level of their Tx (transmitting) and Rx (receiving) elements. As a consequence, their use for diversity gain and spatial multiplexing in a multipleinput multiple-output (MIMO) links [29]-[32] is inherently

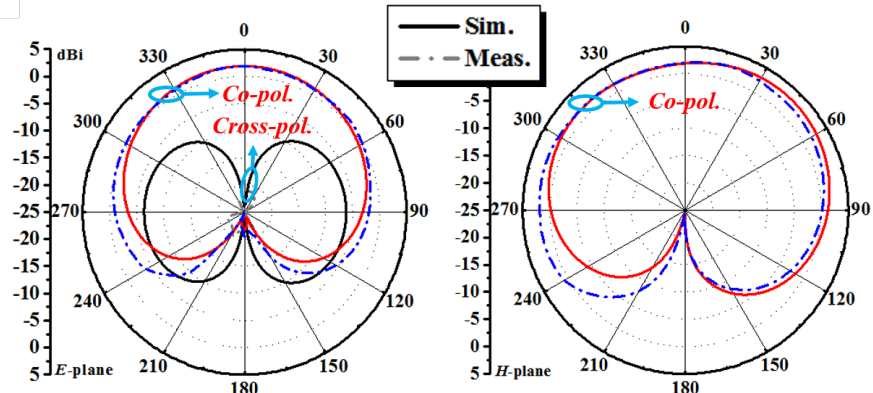

(a)

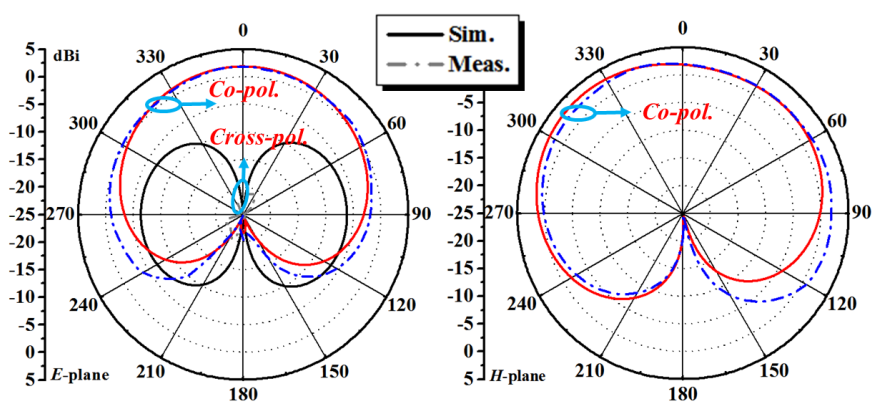

(b)

Fig. 10. Measured (simulated) realized gain patterns of the HDA array with its custom-designed decoupling element in the $E(z 0 x)$ and $H(z 0 y)$ planes at the measured (simulated) resonant frequency. (a) Only Port 1 is excited. (b) Only Port 2 is excited.

limited as was emphasized in [33]. As noted previously, the polarization of the LP Tx and Rx elements of our prototype are parallel. Nevertheless, it achieved a comparative isolation level. Moreover, the developed prototype IBFD system has the desirable advantages of being compact in size and having broadside radiation patterns. They further make it an attractive, competitive candidate for the space-limited applications associated with the previously reported narrow bandwidth IBFD systems [29]-[32].

Because each HDA element's electromagnetic response will remain the same on transmit or receive, the experimentally confirmed performance of the prototype array makes it an ideal candidate for IBFD applications. With the extreme isolation between them, either element could act as the $\mathrm{Tx}$ or $\mathrm{Rx}$ antenna for an IBFD system at the same time. On the other hand, if more directivity was desired than that available from each electrically small HDA alone, the array system could be adapted to perform full Tx or Rx assignments with both HDAs operating at different times. Thus, it could also easily act as a half-duplex system. This flexibility further enhances the attractiveness of the developed compact two-element HDA array.

Performance comparisons of the prototype with recently reported two-element arrays with mutual decoupling structures are given in Table IV. In contrast to the most reported twoelement arrays with an element separation of no less than $0.5 \lambda_{0}$ [13]-[17], our prototype realized a much higher peak isolation level $(50.4 \mathrm{~dB})$ even with a much closer inter-element distance (about $40 \%$ smaller). While the peak realized gain may be lower than the values reported in [18], [20] and 
TABLE IV

Performance Comparison of the Prototype with Recently Reported Two-Element Arrays with Decoupling Structures

\begin{tabular}{|c|c|c|c|c|c|c|c|}
\hline Refs. & $\begin{array}{c}\text { Element Separation } \\
\left(\lambda_{0}\right)\end{array}$ & Array Size $\left(\lambda_{0}^{2}\right)$ & Profile $\left(\lambda_{0}\right)$ & $\begin{array}{c}\text { Peak Realized } \\
\text { Gain }(\mathrm{dBi})\end{array}$ & FTBR $(\mathrm{dB})$ & $\begin{array}{c}\text { Peak Isolation } \\
(\mathrm{dB})\end{array}$ & $\begin{array}{c}\text { Ground Plane } \\
\text { Required }\end{array}$ \\
\hline$[14]$ & 0.5 & $0.99 \times 0.51$ & 0.05 & - & - & 29.0 & Yes \\
\hline$[16]$ & 0.5 & $7.57 \times 4.93$ & 0.254 & - & 9.6 & 38.0 & Yes \\
\hline$[18]$ & 0.58 & $0.70 \times 0.58$ & 0.03 & 5 & 17.6 & 42.0 & Yes \\
\hline$[20]$ & 0.42 & $1.14 \times 0.68$ & 0.021 & 5.9 & 10.0 & 28.3 & Yes \\
\hline$[21]$ & 0.41 & $0.65 \times 0.41$ & 0.013 & 4.12 & - & 25.0 & Yes \\
\hline$[22]$ & 0.35 & $1.48 \times 1.25$ & 0.014 & - & 22.0 & 20.0 & Yes \\
\hline$[24]$ & 0.16 & $0.7 \times 0.5$ & 0.0067 & - & 0.0 & 41.0 & Yes \\
\hline $\begin{array}{c}\text { This } \\
\text { work }\end{array}$ & 0.3 & $\pi \times 0.09$ & 0.049 & 3.10 & 23.0 & 50.4 & No \\
\hline
\end{tabular}

TABLE V

Performance Comparison of the Prototype with Recently Reported Narrowband Antennas ApPlied in IBFD Applications

\begin{tabular}{|c|c|c|c|c|c|c|c|c|}
\hline Refs. & Platform & $\begin{array}{c}\text { Operating } \\
\text { Bandwidth }\end{array}$ & $\begin{array}{c}\text { Antenna Volume } \\
\left(\lambda_{0}{ }^{3}\right)\end{array}$ & $\begin{array}{c}\text { Parallel or } \\
\text { Orthogonal } \\
\text { Polarization }\end{array}$ & $\begin{array}{c}\text { Directional } \\
\text { Radiation }\end{array}$ & $\begin{array}{c}\text { Minimum } \\
\text { Isolation } \\
\text { Level } \\
(\mathrm{dB})\end{array}$ & $\begin{array}{c}\text { Maximum } \\
\text { Isolation } \\
\text { Level } \\
(\mathrm{dB})\end{array}$ & $\begin{array}{c}\text { Average } \\
\text { Isolation } \\
\text { Level }(\mathrm{dB})\end{array}$ \\
\hline$[29]$ & $\begin{array}{c}\text { Handheld radio } \\
\text { system }\end{array}$ & $5.6 \%$ & $\begin{array}{c}0.40 \times 0.27 \times \\
0.385=0.041\end{array}$ & Orthogonal & No & 30 & 33 & $\sim 32$ \\
\hline$[30]$ & $\begin{array}{c}\text { Handheld radio } \\
\text { system }\end{array}$ & $0.92 \%$ & $\begin{array}{c}0.15 \times 0.3 \times 0.02 \\
=0.0009\end{array}$ & Orthogonal & No & 27 & 60 & $\sim 40$ \\
\hline$[31]$ & $\begin{array}{c}\text { Wearable } \\
\text { application }\end{array}$ & $3.3 \%$ & $\begin{array}{c}0.4 \times 0.4 \times 0.016 \\
=0.0025\end{array}$ & Orthogonal & Yes & 25 & 35 & $\sim 30$ \\
\hline$[32]$ & UAV system & $1.7 \%$ & $\begin{array}{c}12 \times 2 \times 0.06= \\
1.44\end{array}$ & Orthogonal & Yes & 40 & 41 & $\sim 41$ \\
\hline $\begin{array}{c}\text { This } \\
\text { work }\end{array}$ & - & $0.66 \%$ & $\begin{array}{c}\pi \times 0.09 \times 0.049 \\
=0.014\end{array}$ & Parallel & Yes & 26 & 50.4 & $\sim 35$ \\
\hline
\end{tabular}

[21], our prototype is one of the smallest array-sized systems. Moreover, while very compact array sizes were also achieved in references [21], [24], their broadside radiation performance is dependent on the presence of a ground plane.

Finally, it is noted that the recently reported HDA system [37] is fundamentally different from the dense two-element array IBFD system developed herein. It is an eight layer, dual circularly polarized (CP), two-port design in which a pair of HDA-based NFRP elements are interleaved into a single structure and driven by a co-located set of tailored driven dipole elements. Because it is a left-hand circularly polarized (LHCP) and right-hand circularly polarized (RHCP) integrated system, it takes advantage of this inherent orthogonal polarization property to achieve the attained high isolation between its ports. Consequently, there is no need for a decoupling element. In distinct contrast, the decoupling element is an intrinsic feature of the dense two-element HDA array developed herein that leads to its reported performance characteristics which are quite attractive for IBFD applications.

\section{CONCLUSION}

A decoupling element was developed and integrated with a broadside radiating, two-element HDA array system for IBFD applications. This custom-designed decoupling element, a pair of face-to-face meander-line resonators connected by a rectangular strip, was introduced on a layer placed on top of the two-element Huygens dipole array. The composite system is compact and low-profile. An equivalent set of electric and magnetic dipoles was introduced to model and explain its operational mechanisms. The analysis of this model was compared with simulation results to verify its efficacy. It was demonstrated that not only did the decoupling element increase the isolation between the ports of the two HDAs to approximately a $50.4 \mathrm{~dB}$ peak level for the very small distance of separation, $0.3 \lambda_{0}$, between them; but it also improved the peak realized gain and FTBR values in comparison to the array without the decoupling element being present. A prototype was fabricated and measured. The measured results, in very good agreement with their simulated values confirmed these attractive performance characteristics. The measured realized gain patterns exhibited very good cardioid shapes and had very wide half-power beamwidths exceeding those of a single HDA.

The operational frequency of the developed IBFD antenna system can be readily shifted to other frequencies of interest. In particular, the operating frequency of previously developed HDA systems have been successfully demonstrated in several different bands. With the reported design process the decoupling element can be similarly reengineered for them as well. The associated attractive performance characteristics of the resulting compact, dense two-element HDA array may prove to be very beneficial, for example, for many narrow bandwidth, space-limited IBFD platforms, e.g., handheld radio systems [29], [30]; systems for wearable applications [31]; and unmanned aerial vehicle (UAV) systems [32]. 


\section{REFERENCES}

[1] S. R. Best, "Progress in the design and realization of an electrically small Huygens source," in Proc. Int. Workshop Antenna Technol. (iWAT), Lisbon, Portugal, pp. 1-4, Mar. 2010,

[2] P. Alitalo, A. O. Karilainen, T. Niemi, C. R. Simovski, and S. A. Tretyakov, "Design and realisation of an electrically small Huygens source for circular polarisation," IET Microw., Antennas Propag.,vol.5, no. 7, pp. 783-789, May 2011.

[3] T. Niemi, P. Alitalo, A. O. Karilainen, and S. A. Tretyakov, "Electrically small Huygens source antenna for linear polarisation," IET Microw. Antennas Propag.,vol. 6, no. 7, pp. 735-739, 2012.

[4] R. W. Ziolkowski, "Low profile, broadside radiating, electrically small Huygens source antennas," IEEE Access, vol. 3, pp. 2644--2651, 2015.

[5] M.-C. Tang, Z. Wu, T. Shi, H. Zeng, W. Lin and R. W. Ziolkowski, "Duallinearly polarized, electrically small, low-profile, broadside radiating, Huygens dipole antenna," IEEE Trans. Antennas Propag.,vol. 66, no. 8, pp. 3877-3885, Aug. 2018.

[6] M.-C. Tang, Z. Wu, T. Shi, and R. W. Ziolkowski, "Electrically small, low-profile, planar, Huygens dipole antenna with quad-polarization diversity," IEEE Trans. Antennas Propag., vol. 66, no. 12, pp. 6772-6780, Dec. 2018.

[7] Z. Wu, M.-C. Tang, M. Li, and R. W. Ziolkowski, "Ultralow-profile, electrically small, pattern-reconfigurable metamaterial-inspired Huygens dipole antenna," IEEE Trans. Antennas Propag.,vol. 68, no. 3, pp. 1238 1248, Mar. 2020.

[8] M.-C. Tang, T. Shi, and R. W. Ziolkowski, "Electrically small, broadside radiating Huygens source antenna augmented with internal non-Foster elements to increase its bandwidth," IEEE Antennas Wireless Propag. Lett., vol. 16, pp. 712-715, 2017.

[9] A. Sabharwal, P. Schniter, D. Guo, D. W. Bliss, S. Rangarajan and R. Wichman, "In-band full-duplex wireless: Challenges and opportunities," IEEE J. Sel. Areas Commun., vol. 32, no. 9, pp. 1637-1652, Sept. 2014.

[10] T.Le-Ngoc and A.Masmoudi, Full-Duplex Wireless Communications Systems: Self-interference Cancellation. Berlin, Germany: Springer, 2017.

[11] D. Bharadia, E. McMilin, and S. Katti, "Full duplex radios," in Proc. Int. Conf. ACM SIGCOMM, New York, NY, USA, 12-16 August 2013, pp. $375-386$

[12] H. Nawaz and I. Tekin, "Dual-polarized, differential fed microstrip patch antennas with very high interport isolation for full-duplex communication," IEEE Trans. Antennas Propag., vol. 65, no. 12, pp. 7355-7360, Dec. 2017.

[13] M.-C. Tang, Z. Chen, W. Hao, M. Li, B. Luo, J. Wang, Z. Shi, and R. W. Ziolkowski, "Mutual coupling reduction using meta-structures for wideband, dual-polarized, and high-density patch arrays," IEEE Trans. Antennas Propag., vol. 65, no. 8, pp. 3986-3998, Aug. 2017.

[14] M. Coulombe, S. F. Koodiani, and C. Caloz, "Compact elongated mushroom (EM)-EBG structure for enhancement of patch antenna array performances," IEEE Trans. Antennas Propag., vol. 58, no. 4, pp. 10761086, Apr. 2010.

[15] S. Xiao, M.-C. Tang, Y.-Y. Bai, S. Gao, and B.-Z. Wang, "Mutual coupling suppression in microstrip array using defected ground structure," IET Microw. Antennas Propag., vol. 5, no. 12, pp. 1488-1494, May. 2011.

[16] M. Farahani, J. Pourahmadazar, M. Akbari, M. Nedil, A. R. Sebak, and T. A. Denidni, "Mutual coupling reduction in millimeter-wave MIMO antenna array using a metamaterial polarization-rotator wall," IEEE Antennas Wireless Propag. Lett., vol. 16, pp. 2324-2327, 2017.

[17] K. L. Chung, and S. Kharkovsky, "Mutual coupling reduction and gain enhancement using angular offset elements in circularly polarized patch array," IEEE Antennas Wireless Propag. Lett., vol. 12, pp. 1122-1124, 2013.

[18] X. Sun and M. Y. Cao, "Low mutual coupling antenna for WLAN application," Electron. Lett., vol.53, no. 6, pp. 368-370, Mar. 2017.

[19] K.-L Wu, C. Wei, X. D. Mei, and Z.-Y. Zhang, "Array-antenna decoupling surface," IEEE Trans. Antennas Propag., vol. 65, no. 12, pp. 6728-6738, Dec. 2017.

[20] B. L. Dhevi, K. S. Vishvaksenan, and K. Rajakani, "Isolation enhancement in dual-band microstrip antenna array using asymmetric loop resonator," IEEE Antennas Wireless Propag. Lett., vol. 17, no. 2, pp. 238-241, Feb. 2018.

[21] X. M. Ling, and R. L. Li, "A novel dual-band MIMO antenna array with low mutual coupling for portable wireless devices," IEEE Antennas Wireless Propag. Lett., vol. 10, pp. 1039-1042, 2011.

[22] X. M. Yang, X. G. Liu, X.Y. Zhou, and T. J. Cui,, "Reduction of mutual coupling between closely packed patch antennas using waveguided metamaterials," IEEE Antennas Wireless Propag. Lett., vol. 11, pp. 389391, 2012.

[23] S. Zhang, and G. F. Pedersen, "Mutual coupling reduction for UWB MIMO antennas with a wideband neutralization line," IEEE Antennas Wireless Propag. Lett., vol. 15, pp. 166-169, 2016.

[24] C.-D. Xue, X. Y. Zhang, Y. F. Cao, Z. Hou, and C. F. Ding, "MIMO antenna using hybrid electric and magnetic coupling for isolation enhancement," IEEE Trans. Antennas Propag., vol. 65, no. 10, pp. 5162 5170 , Oct. 2017.

[25] M.-C. Tang, H. Wang, and R. W. Ziolkowski, "Design and testing of simple, electrically small, low-profile, Huygens source antennas with broadside radiation performance," IEEE Trans. Antennas Propag., vol. 64, no. 11, pp. 4607-4617, Nov. 2016.

[26] C. A. Balanis, Antenna Theory: Analysis and Design., John Wiley \& Sons, 2016.

[27] C. Icheln, J. Krogerus, and P. Vainikainen, "Use of balun chokes in small-antenna radiation measurements," IEEE Trans. Instrum. Meas., vol. 53, no. 2, pp. 498-506, Apr. 2004.

[28] X. Lin, Z. Xie, P. Zhang, and Y. Zhang, "A broadband filtering duplex patch antenna with high isolation," IEEE Antennas Wireless Propag. Lett., vol. 16, pp. 1937-1940, 2017.

[29] A. H. Abdelrahman and D. S. Filipovic, "Antenna system for full-duplex operation of handheld radios," IEEE Trans. Antennas Propag., vol. 67, no. 1 , pp. 522-530, Jan. 2019.

[30] O. N. Alrabadi, A. D. Tatomirescu, M. B. Knudsen, M. Pelosi, and G. F. Pedersen, "Breaking the transmitter-receiver isolation barrier in mobile handsets with spatial duplexing," IEEE Trans. Antennas Propag., vol. 61, no. 4, pp. 2241-2251, Apr. 2013.

[31] C. X. Mao, Y. Zhou, Y. Wu, H. Soewardiman, D. H. Werner and J. S. Jur, "Low-profile strip-loaded textile antenna with enhanced bandwidth and isolation for full-duplex wearable applications," IEEE Trans. Antennas Propag., vol. 68, no. 9, pp. 6527-6537, Sept. 2020.

[32] A. Samaiyar, A. H. Abdelrahman and D. S. Filipovic, "Simultaneous transmit and receive reflectarray antennas on low cost UAV platforms," in 2017 IEEE International Symposium on Antennas and Propagation \& USNC/URSI National Radio Science Meeting., San Diego, CA, 2017, pp. 2047-2048.

[33] K. Iwamoto, M. Heino, K. Haneda and H. Morikawa, "Design of an antenna decoupling structure for an inband full-duplex collinear dipole array," IEEE Trans. Antennas Propag., vol. 66, no. 7, pp. 3763-3768, Jul. 2018.

[34] F. Yang and Y. Rahmat-Samii, "Microstrip antennas integrated with electromagnetic band-gap (EBG) structures: A low mutual coupling design for array applications," IEEE Trans. Antennas Propag., vol. 51, no. 10, pp. 2936-2946, Oct. 2003.

[35] C. A. Balanis, Antenna Theory: Analysis and Design, 3rd ed. Hoboken, NJ, USA: Wiley Interscience, 2005, Chap. 8.

[36] R. F. Harrington, "On the gain and beamwidth of directional antennas," IRE Trans. Antennas Propag., vol. 6, no. 3, pp. 219--225, Jul. 1958.

[37] Z. Wu, M.-C. Tang, T. Shi and R. W. Ziolkowski, "Two-port, dualcircularly polarized, low-profile broadside-radiating electrically small Huygens dipole antenna," IEEE Trans. Antennas Propag., early access, doi:10.1109/TAP.2020.2999747, Jun. 2020. 


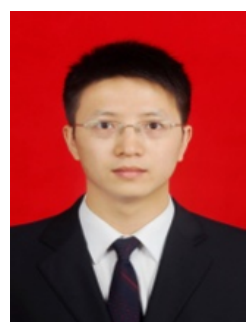

Ming-Chun Tang (Senior Member, IEEE) received the B. S. degree in physics from the Neijiang Normal University, Neijiang, China, in 2005 and the Ph. D. degree in radio physics from the University of Electronic Science and Technology of China (UESTC), in 2013. From August 2011 to August 2012, he was also with the Department of Electrical and Computer Engineering, The University of Arizona, Tucson, AZ, USA, as a Visiting Scholar. He is currently a full Professor in the School of Microelectronics and Communication Engineering, Chongqing University, China. His research interests include electrically small antennas, RF circuits, metamaterial designs and their applications.

Prof. Tang is the Senior Member of the Chinese Institute of Electronics. $\mathrm{He}$ was a recipient of the National Science Fund for Excellent Young Scholars in 2019. He was a recipient of the Best Student Paper Award in the 2010 International Symposium on Signals, Systems and Electronics (ISSSE2010) held in Nanjing, China. His Ph.D. students received Best Student Paper Awards from the IEEE 7th Asia-Pacific Conference on Antennas and Propagation (2018 IEEE APCAP) held in Auckland, New Zealand, 2019 IEEE International Applied Computational Electromagnetics Society (ACES) Symposium held in Nanjing, China, 2019 IEEE International Workshop on Electromagnetics: Applications and Student Innovation Competition held in Qingdao, China, and 2019 Cross Strait Quad-Regional Radio Science and Wireless Technology Conference held in Taiyuan, China. He is the founding Chair of the IEEE AP-S / MTT-S Joint Chongqing Chapter. He serves on the Editorial Boards of several journals, including IEEE Access, Electronics Letters and IET Microwaves, Antennas \& Propagation. He has also served on the review boards of various technical journals, and many international conferences as a General Chair, TPC Member, Session Organizer, and the Session Chair.

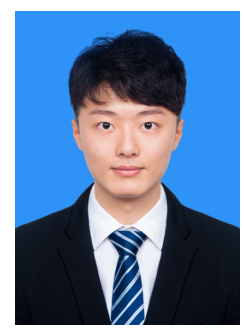

Hanwen Tu received the B.S. degree from the Liaoning University of Science and Technology, Liaoning, China, in 2017. He is currently pursuing the M.S. degree in electronic communication engineering with Chongqing University, Chongqing, China.

His current research interests include Huygens dipole antenna and decoupling research of arrays.

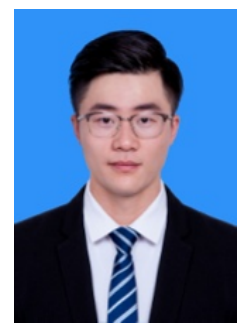

Zhentian Wu (Student Member, IEEE) was born in Hefei, China, in 1994. He received the B.S. degree from the West Anhui University, Luan, China, in 2016. He is currently pursuing the ph. D degree in electronics and communication engineering in the School of Microelectronics Communication Engineering, Chongqing University, China.

His current research interests include electrically small antenna and directional antenna and their applications.

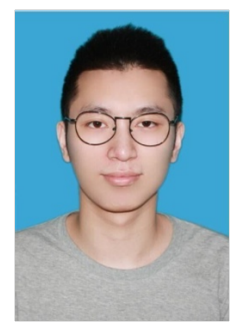

Xiaoming Chen (Student Member, IEEE) received the B.S. degree in electronics and information engineering from Chongqing University, Chongqing, China, in 2016, where he is currently pursuing the $\mathrm{Ph} . \mathrm{D}$. degree in information and communication technology engineering with the School of Microelectronics and Communication Engineering.

His current research interests include intelligent algorithms in antenna design and circularly polarized antennas.

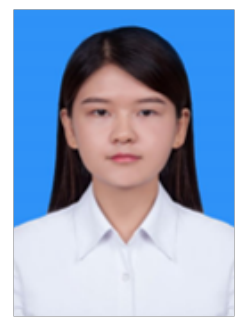

Ting Shi (Student Member, IEEE) received the B.S. degree from the University of Electronic Science and Technology of China (UESTC), Chengdu, China, in 2014, the M.S. and Ph.D. degrees from Chongqing University, Chongqing, China, in 2017 and 2020. She is currently a Postdoctoral Fellow with the School of Electronic Science and Engineering (National Exemplary School of Microelectronics), UESTC

Her current research interests include microwave antennas and metasurfaces.

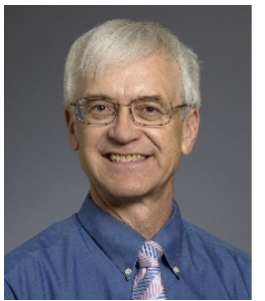

Richard W. Ziolkowski received the B.Sc. (magna cum laude) degree (Hons.) in physics from Brown University, Providence, RI, USA, in 1974; the M.S. and $\mathrm{Ph} . \mathrm{D}$. degrees in physics from the University of Illinois at Urbana-Champaign, Urbana, IL, USA, in 1975 and 1980, respectively; and the Honorary Doctorate degree from the Technical University of Denmark, Kongens Lyngby, Denmark in 2012.

$\mathrm{He}$ is currently a Distinguished Professor in the Global Big Data Technologies Centre in the Faculty of Engineering and Information Technologies (FEIT) at the University of Technology Sydney, Ultimo NSW, Australia. He became a Professor Emeritus at the University of Arizona in 2018, where he was a Litton Industries John M. Leonis Distinguished Professor in the Department of Electrical and Computer Engineering in the College of Engineering and was also a Professor in the College of Optical Sciences. He was the Computational Electronics and Electromagnetics Thrust Area Leader with the Lawrence Livermore National Laboratory, Engineering Research Division, in Livermore, CA before joining The University of Arizona, Tucson, AZ, USA, in 1990. His current research interests include the application of new mathematical and numerical methods to linear and nonlinear problems dealing with the interaction of electromagnetic and acoustic waves with complex linear and nonlinear media, as well as metamaterials, metamaterial-inspired structures, nano-structures, and other classical and quantum applicationsspecific configurations.

Prof. Ziolkowski became a Fellow of the Optical Society of America in 2006 and the American Physical Society in 2016. He was the recipient of the 2019 IEEE Electromagnetics Award (IEEE Field Award). He was the Australian DSTO Fulbright Distinguished Chair in Advanced Science and Technology from 2014-2015. He was a 2014 Thomas-Reuters Highly Cited Researcher. He served as the President of the IEEE Antennas and Propagation Society in 2005 . He is also actively involved with the URSI, EurAAP, OSA and SPIE professional societies. 